\title{
A REUSABLE IN-PLANE POLYMER INTEGRATED MICROFLUIDIC INTERCONNECT
}

\author{
Ronalee Lo and Ellis Meng \\ Department of Biomedical Engineering, University of Southern California, Los Angeles, USA \\ (Tel : +1-213-740-6952; E-mail: rlo@usc.edu)
}

\begin{abstract}
A novel reusable, in-plane polymer interconnect directly integrated into a microfluidic system is demonstrated. This approach features plug-and-play style connections to microfluidic networks in which commercially available non-coring syringe needles are simply inserted through pre-defined polydimethylsiloxane (PDMS) septums to form rapid, on-demand connections to microchannels. These septums are mechanically anchored by one layer of SU-8 that also forms the microchannels. Interconnect design, fabrication, theoretical analysis, and experimental results (pull-out force and leakage) are presented.
\end{abstract}

Keywords: microfluidic packaging, microfluidic interconnects, septum

\section{INTRODUCTION}

Macro-to-microfluidic connections and packaging is an ongoing challenge despite many efforts [1-3]. Current microfluidic packaging strategies focus on out-of-plane connection formats. These existing methodologies have not found widespread adoption due to limitations related to complexity in application (i.e. requiring labor-intensive precision alignment), use of adhesives, and/or extra fabrication steps. A tradeoff commonly encountered in these interconnects is the dramatic increase in dead volume and space necessitated by the spatial separation required for magnified viewing without obstructing microscope objectives [4]. Our approach features an in-plane microfluidic interconnect format and seeks to overcome these challenges to provide a high yield, low cost, rapid, and convenient method for realizing macro-to-microfluidic connections.

Our interconnection and packaging method is inspired by the reusable microelectronics plug-in format (pin-and-socket). A small diameter syringe needle corresponds to the "pin" and the "socket" is formed by a PDMS septum housed in an SU-8 anchoring structure that is either part of a microchannel or connected to one. The needle is inserted through the PDMS septum and SU-8 anchor to form a fluidic connection (Fig. 1-2). The SU-8 anchor also serves as a needle insertion guide. These connections are formed rapidly and on-demand without the need for adhesives, precise alignment, thru-wafer drilling/etching, or complex post-processing/assembly. Also, due to the selfsealing nature of silicone rubber, syringe needles can be removed and interchanged with another needle [5]. These interconnects are batch fabricated, maximize usable microfluidic real estate, and are easily integrated with other microfluidic elements such as electrolysis pumps and flow sensors.

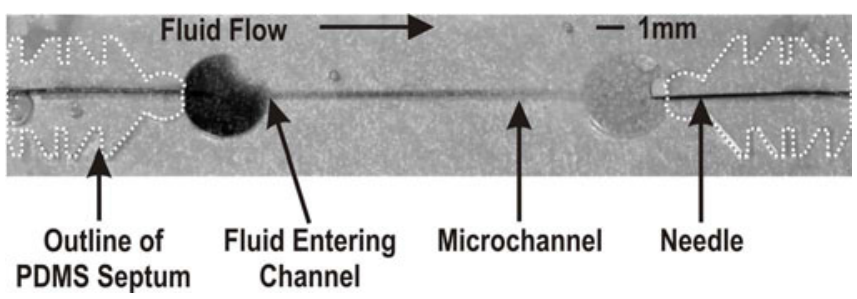

Fig. 1 Image of interconnect with needles piercing both input and output PDMS septums; the PDMS septums are outlined to show their location. Dyed fluid is being introduced via the inserted needle.

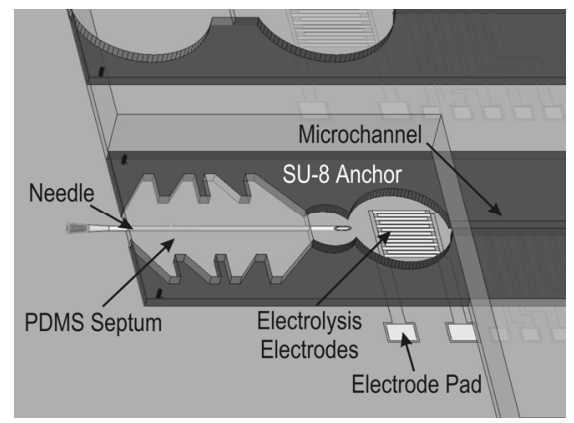

Fig. $23 D$ illustration of the integrated microfluidic interconnect concept. 
This approach is demonstrated using a single layer of SU-8 to define the mechanical anchor for the PDMS septum, needle alignment guide, and microchannel. Three different SU-8 septum anchor shapes (square, circular, and barbed) were examined (Fig. 3). The SU-8 anchor structures can also be fabricated on top of surface micromachined channels and combined with PDMS septums to form microfluidic interconnects. This process requires just one additional mask layer to incorporate batch fabricated interconnect structures.

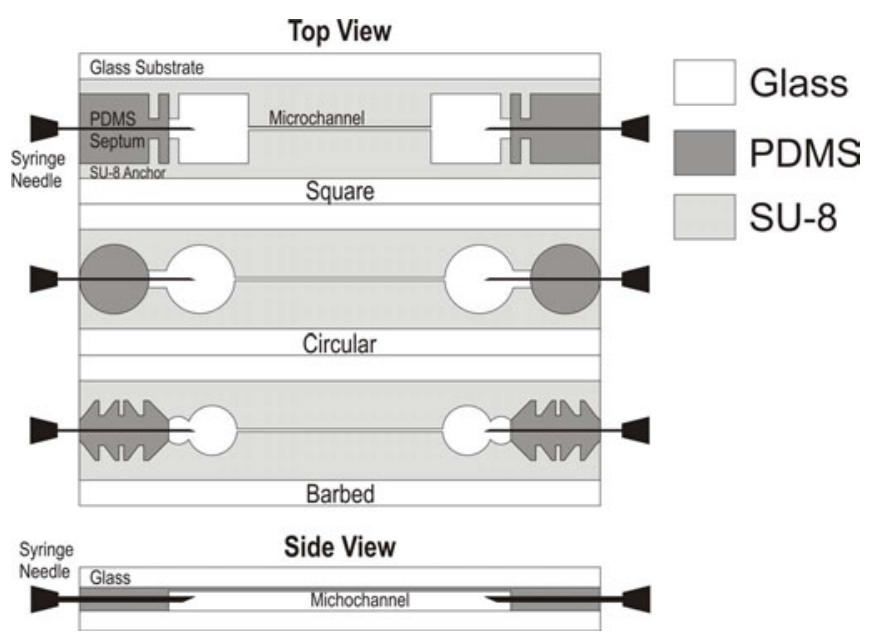

Fig. 3 Illustration of the 3 interconnect designs.

\section{FABRICATION}

Standard MEMS processes are used to fabricate the interconnects to facilitate integration with commonly used microfluidic designs. AZ 4400 photoresist was spun onto a glass substrate and patterned to define electrolysis electrodes and resistive thermal flow sensors (Fig. 4A). $200 \AA$ of $\mathrm{Ti}$ and then $2000 \AA$ of $\mathrm{Pt}$ was deposited by e-beam evaporation. The electrodes and sensors were defined by liftoff (Fig. 4B). Excess metal was removed by gently brushing the surface with a cleanroom swab or with the aid of sonication. Parylene C, an inert and biocompatible polymer, was vapor deposited onto the surface (Fig. 4C); Parylene $\mathrm{C}$ serves as an insulation layer for the electrodes and sensor traces. A second lithography step is performed and parylene $\mathrm{C}$ is removed by reactive ion etching in $\mathrm{O}_{2}$ plasma to expose the electrolysis electrodes and the contact

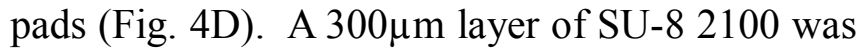

spun and patterned to form the microchannel walls, mechanical anchor for the PDMS septum, and needle alignment guide. The area defining the interconnect was filled with PDMS (Sylgard 184) in order to create the PDMS septum (Fig. 4E). Water is used as a mask to prevent the spread of PDMS into the microchannel or electrolysis microchamber during the septum forming step. The entire system is then sealed with a PDMS coated glass slide (Fig. 4F) which caps the microchannel and completes the integrated interconnect (Fig. 5).
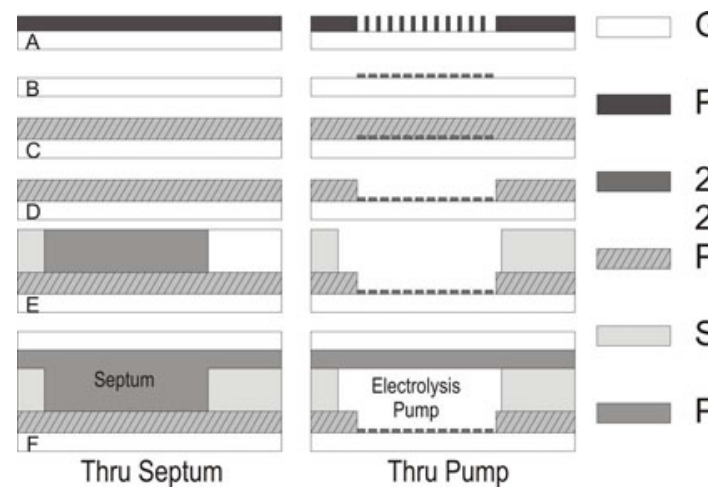

Glass

Photoresist

$200 \AA$ Ti/ $2000 \AA \mathrm{Pt}$ Parylene

SU-8 2100

Fig. 4 Fabrication steps for the microfluidic system shown (left) through the septum and (right) through the electrolysis pump.

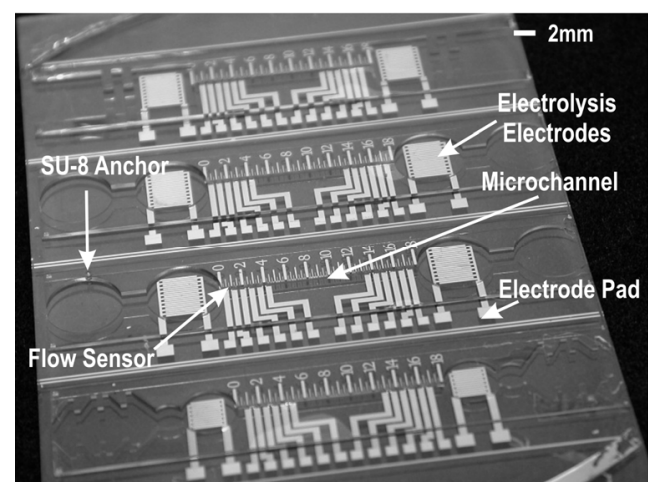

Fig. 5 Fabricated microfluidic system with integrated interconnect, electrolysis pump, micro reservoir, flow sensors, and microchannel. PDMS septums and microchannel cap not shown.

\section{EXPERIMENTS AND DISCUSSION}

\subsection{Pull-out Test}

The theoretical mechanical robustness of the interconnect scheme is determined by examining pull-out force. The system is modeled as stiff 
fiber (needle) in a soft matrix (PDMS septum). Total pull-out force is the sum of frictional force and adhesion strength between the fiber and matrix:

$$
F=2 \pi r k X+F_{0}
$$

where $r$ is the fiber radius, $k=\mu p$, which is constant during debonding ( $\mu$ is the coefficient of friction and $p$ is the compressive stress), and $X$ is the debonded length. For the case where frictional force is absent, $X=0$, the equation simplifies to the pull-out force due to adhesion, $F_{0} . \quad F_{0}$ is defined by the Griffith's energy criterion:

$$
F_{0}^{2}=4 \pi A r E_{m} G_{a}
$$

$A$ is the cross-sectional area of the matrix, $r$ is the fiber radius, $E_{m}$ and $G_{a}$ are the Young's modulus and fracture energy of the matrix, respectively [6].

The pull-out force was obtained experimentally by inserting a 33 gauge luer lock stainless steel needle into the PDMS septum and increasing weight applied to the needle until the needle was pulled out (Fig. 6). In order to get optimal sealing after the needle is removed, a non-coring needle is used to pierce the septum because it only displaces the material as the needle is inserted. A coring needle removes material as it is inserted into the PDMS matrix; which can clog the needle and lead to leakage after the needle is removed [5].

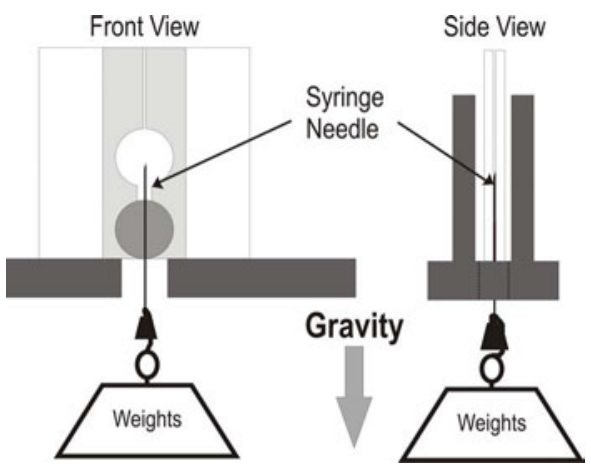

Fig. 6 Test setup to measure the pull-out force necessary to remove the needle from the PDMS septum.

Repeated trials were performed on the same interconnect ( $\mathrm{N}=6$ for each design). The pull-out force was observed to be highest for the first pull- out, then decrease over subsequent trials until it reached a steady state value over multiple pullouts. Pull-out force for the first trial is consistent with theoretical values (Fig. 7). A comparison of interconnects found in literature that have reported repeated pull-out trials is shown in Fig. 8. As predicted in the theoretical equations, contact area, which is dependant on the contact length, between the interconnect and needle plays a significant role in determining pull-out force.

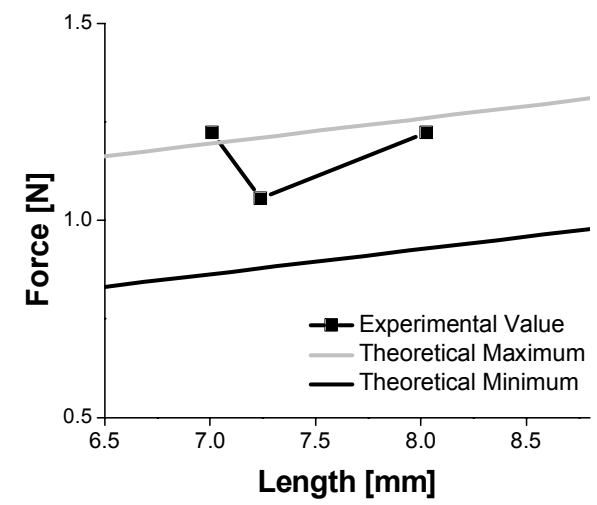

Fig. 7 Pull-out force for the first needle removal. The theoretical maximum and minimum pull-out forces, calculated from Eq. (1), are shown.

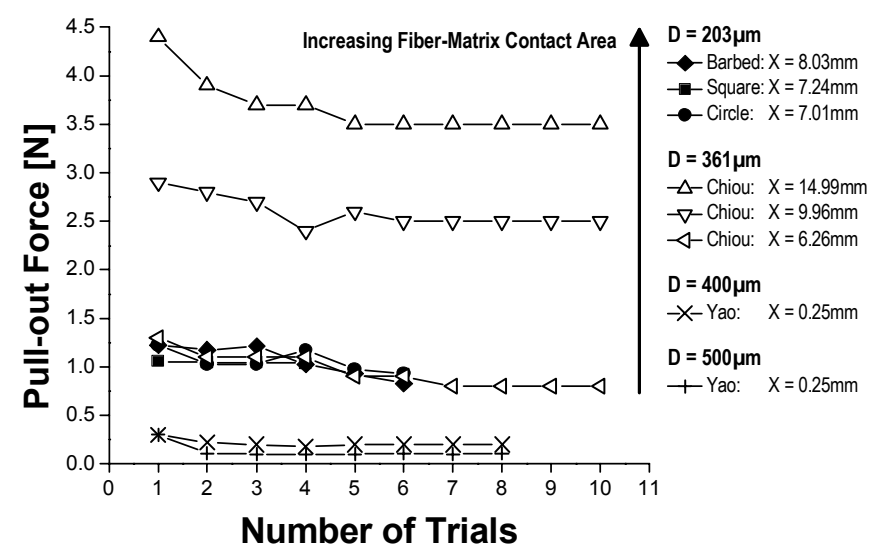

Fig. 8 Comparison of pull-out force of our connectors and others [7, 8].

\subsection{Leakage Pressure Test}

Interconnect operating range was obtained by pressurizing with water or $\mathrm{N}_{2}$ gas. 33 gauge luer lock needles were inserted into input and output interconnects of a system. The output luer lock needle was blocked with a plug (Fig. 9). 


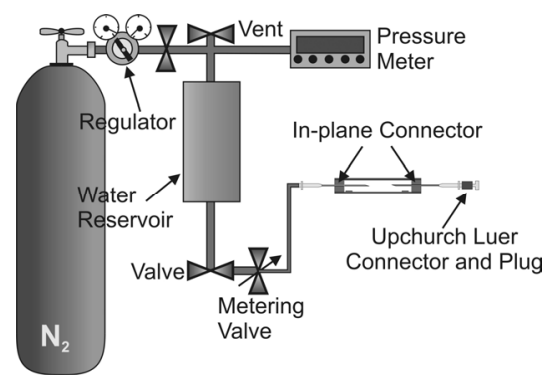

Fig. 9 Pressure test setup.

The input side was pressurized and the pressure at which leakage occurred, site of leakage, and failure mechanism were recorded (Table 1).

Table 1 Leakage pressure results

\begin{tabular}{|c|c|c|c|}
\hline Type & $\begin{array}{c}\text { Test } \\
\text { Fluid }\end{array}$ & $\begin{array}{c}\text { Leakage } \\
\text { Pressure } \\
{[\mathbf{k P a}]}\end{array}$ & Leakage Location \\
\hline Square & Water & 20.82 & Glass/parylene interface \\
\hline Circle & Water & 36.27 & Glass/parylene interface \\
\hline Barbed & Water & 51.37 & Output needle insertion \\
\hline Square & N2 & 25.72 & Output needle insertion \\
\hline Circle & N2 & 60.4 & Glass/parylene interface \\
\hline Barbed & N2 & 15.72 & Glass/parylene interface \\
\hline
\end{tabular}

Manufacturing variation may have contributed to the large range of leakage pressures. Failure of the glass-parylene interface either by delamination of the parylene from the glass substrate, or the needle puncturing the parylene layer and becoming embedded under the parylene, limited the ability to test the operating range of the connector under pressure. Subsequent interconnect test structures used adhesion promoter A174 prior to parylene deposition to enhance adhesion at the parylene-glass interface.

\subsection{Prolonged Pressure Test}

Constant water pressure was applied to the interconnects to evaluate the long term durability of the connections. The setup was identical to the setup used in the leakage pressure test. $36.2 \mathrm{kPa}$ was applied to the input needle of a circular port connector for a period of 24 hours. There was no measurable pressure drop or leakage observed over this time period.

\section{CONCLUSION}

A novel microfluidic interconnect method features rapid, on-demand connections to microfluidic devices is demonstrated. These connectors leverage the sealing nature of PDMS to form simple connections to microfluidics with commercially available syringe needles.

\section{ACKNOWLEDGEMENTS}

This work was funded in part by the Engineering Research Centers Program of the NSF (EEC-0310723) and an NSF CAREER grant (EEC-0547544). The authors would like to thank Mr. Merrill Roragen, Dr. Tuan Hoang, and members of the Biomedical Microsystem Lab at the University of Southern California.

\section{REFERENCES}

[1] A. Puntambekar and C. H. Ahn, "Selfaligning microfluidic interconnects for glassand plastic-based microfluidic systems," $J$. Micromech. Microeng., vol. 12, pp. 35-40, 2002.

[2] A. V. Pattekar and M. V. Kothare, "Novel microfluidic interconnectors for high temperature and pressure applications," $J$. Micromech. Microeng., vol. 13, pp. 337-345, 2003.

[3] S. Matsumoto, J. Xie, and Y. C. Tai, "Polymer micro interface for fluidic probing," presented at $\mu$ TAS, California, USA, 2003.

[4] E. S. Lee, D. Howard, E. Liang, S. D. Collins, and R. L. Smith, "Removable tubing interconnects for glass-based micro-fluidic systems made using ECDM," J. Micromech. Microeng., vol. 14, pp. 535-541, 2004.

[5] R. Lo, K. Kuwahara, P. Y. Li, R. Agrawal, M. S. Humayun, and E. Meng, "A passive refillable intraocular MEMS drug delivery device," Okinawa, Japan, 2006.

[6] A. N. Gent and G. L. Liu, "Pull-out and fragmentation in model fiber composites," $J$. Mat Sci, vol. 26, pp. 2467-2476, 1991.

[7] C. H. Chiou and G. B. Lee, "Minimal deadvolume connectors for microfluidics using PDMS casting techniques," J. Micromech. Microeng., vol. 14, pp. 1484-1490, 2004.

[8] T.-J. Yao, S. Lee, W. Fang, and Y.-C. Tai, "Micromachined rubber O-ring micro-fluidic couplers," Proc. of the IEEE MEMS, pp. 624627, 2000. 